\title{
On the Optimum Demodulation in the Presence of Gaussian Phase Noise
}

\author{
Simon Bicaïs ${ }^{\dagger}$, Jean-Baptiste Doré ${ }^{\dagger}$, José Luis Gonzalez Jimenez ${ }^{\dagger}$ \\ ${ }^{\dagger}$ CEA-Leti, Minatec Campus, Grenoble, France \\ contact: simon.bicais@cea.fr
}

\begin{abstract}
Millimeter-wave systems appears to be one of the foremost solutions to face the exponential growth of the data traffic. Yet, their performance are severely degraded by oscillators phase noise. This paper addresses the design of optimum receivers affected by phase noise. We first derive the optimum decision metric for symbol detection under the assumption of a Gaussian phase noise and a high signal-to-noise ratio. In contrast to state-of-the-art approaches, we propose to represent within a complete metric space the demodulation over the phase noise channel. The provided framework enables the use of efficient algorithms to perform the demodulation. Computable probabilistic demapper values are derived upon the optimum metric to implement soft channel decoding. This work shows that knowledge of the channel statistics can be capitalized to enhance performance of both coded and uncoded systems. Eventually, we propose the necessary channel estimation scheme and evaluate the performance degradation due to estimation errors.
\end{abstract}

Index Terms-Millimeter wave, Phase noise, Maximum likelihood detection, Complete metric space, Channel estimation

\section{INTRODUCTION}

With regard to the spectrum shortage in the cellular bands, the millimeter-wave (mmWave) spectrum has aroused lots of interest in the research domain. With unprecedentedly large bandwidth, mmWave systems are considered to be one of the foremost solutions to face the exponential growth of the data traffic. Within the next couple of years, a requirement of $10+$ Gbit/s user throughput is foreseen. Nonetheless, the poor performance of high frequency oscillators [1] plagues mmWave systems with strong Phase Noise (PN). This has motivated extensive work on the design of optimum receivers for systems impacted by PN. Numerous of these optimum receivers are listed in [2] for the common PN models. Still, most of the literature's detectors present complex structures hindering any analytical study. The development of telecommunications has proved that simple analytical frameworks are fundamental for the design of communications systems as they provide means to pursue comprehensive analyses and thus develop efficient algorithms.

Contributions: Through this work, we aim at improving the design of high-rate mmWave communications. This paper mainly pursues the study of Krishnan [2]. Similarly, we assume a high SNR and a Gaussian PN to derive the already known Maximum-Likelihood (ML) decision premetric for symbol detection. In contrast to [2], we exploit further the high SNR approximation to properly define the polar metric. Our metric achieves the same optimum performance of the $M L$ premetric but enables us to come within the scope of complete metric spaces. Representing signals upon an appropriate metric space allows the use of efficient algorithms to perform the demodulation. Further, to enhance performance of coded systems, we propose computable probabilistic demapper values using the polar metric to implement efficient soft decision decoding. Though knowledge of the channel statistics improves the performance, it requires a channel estimation. Accordingly, we propose ML estimators of the thermal and phase noise variances. The performance degradation due to estimation errors of the polar metric are eventually evaluated and compared to the ML premetric one.

Organization: The remainder of this paper is structured as follows. Section II introduces a brief description of the channel and PN models. Section III derives the polar metric decision rule for optimal symbol detection impacted by Gaussian PN. Further, Section IV is devoted to the evaluation of probabilistic demapper values. Finally, an appropriate channel estimation is proposed in Section V to implement the optimal demodulation.

\section{System Model}

\section{A. Channel}

We consider the complex Additive White Gaussian Noise (AWGN) channel impacted by PN. The received signal is then written as

$$
r=s \cdot e^{j \phi}+n,
$$

where $s$ is the modulated symbol, $\phi$ is the oscillator PN and $n$ represents a zero-mean circular complex Gaussian noise with variance $2 \sigma_{n}^{2}$. The transmitted symbol $s$ belongs to the constellation $\mathcal{C}$ with average energy $E_{s}$ and modulation order $M$. We will use the polar representation such that $s_{\rho}$ and $s_{\theta}$ stand respectively for the amplitude and phase of symbol $s$.

\section{B. Phase noise}

The Gaussian distribution is mathematically convenient and thus often exploited to pursue simple analytical analyses [3]. In fact, it appears to be an appropriate PN model for mmWave systems. When considering wide bandwidth systems, the oscillator noise floor represents the greatest contribution to the overall PN [4]. Then the oscillator PN $\phi$ is to be described by a zero-mean Gaussian distribution with variance $\sigma_{p}^{2}$ as follows

$$
\phi \sim \mathcal{N}\left(0, \sigma_{p}^{2}\right)
$$




\section{MAXimum LiKelihood Symbol Detection}

\section{A. Optimum decision metric at high SNR}

Regarding symbol-by-symbol detection, the Symbol Error Probability (SEP) is minimized by the Maximum A Posteriori (MAP) decision criterion [5]. When transmitted symbols are equiprobable and mutually independent, this criterion simplifies to the ML decision rule [5]. The optimum receiver decision is hence defined upon the likelihood function $p(r \mid s)$. With regard to the channel model in Eq. (1), it is relevant to rewrite

$$
p(r \mid s)=p\left(r_{\rho}, r_{\theta} \mid s_{\rho}, s_{\theta}\right) .
$$

Let us denote by $n^{\prime}$ the shifted noise $n \cdot e^{-j\left(s_{\theta}+\phi\right)}$ which is identically distributed to $n$ as the complex AWGN is circular. We then study the received symbol through its amplitude and phase as

$$
\begin{aligned}
r_{\rho} & =\left|\left(s_{\rho}+n^{\prime}\right) \cdot e^{j\left(s_{\theta}+\phi\right)}\right| \\
& =\sqrt{\left(s_{\rho}+\Re\left(n^{\prime}\right)\right)^{2}+\Im\left(n^{\prime}\right)^{2}} \\
& \simeq s_{\rho}+\Re\left(n^{\prime}\right)
\end{aligned}
$$

and

$$
\begin{aligned}
r_{\theta} & =\arg \left[\left(s_{\rho}+n^{\prime}\right) \cdot e^{j\left(s_{\theta}+\phi\right)}\right] \\
& =s_{\theta}+\phi+\arctan \left(\frac{\Im\left(n^{\prime}\right)}{s_{\rho}+\Re\left(n^{\prime}\right)}\right) \\
& \simeq s_{\theta}+\phi+\frac{\Im\left(n^{\prime}\right)}{s_{\rho}} .
\end{aligned}
$$

These first-order approximations clearly hold under a high SNR assumption. Further, exploiting the high SNR assumption enables to derive a simple closed-form expression of the channel likelihood function [2]. We conclude from the thermal and phase noise model in Eq. (1) and (2) that

$$
\begin{aligned}
r_{\rho} & \sim \mathcal{N}\left(s_{\rho}, \sigma_{n}^{2}\right), \\
r_{\theta} & \sim \mathcal{N}\left(s_{\theta}, \sigma_{p}^{2}+\sigma_{n}^{2} / s_{\rho}^{2}\right) .
\end{aligned}
$$

It is straightforward that the likelihood function follows a bivariate Gaussian distribution defined by

$$
p(r \mid s)=\frac{\exp \left(-\frac{1}{2}\left(\frac{\left(r_{\rho}-s_{\rho}\right)^{2}}{\sigma_{n}^{2}}+\frac{\left(r_{\theta}-s_{\theta}\right)^{2}}{\sigma_{p}^{2}+\sigma_{n}^{2} / s_{\rho}^{2}}\right)\right)}{2 \pi \sqrt{\sigma_{n}^{2}\left(\sigma_{p}^{2}+\sigma_{n}^{2} / s_{\rho}^{2}\right)}} .
$$

This expression has been originally derived in [2] and leads to the ML detection decision at the receiver given by

$$
\begin{aligned}
\hat{s} & =\underset{s \in \mathcal{C}}{\arg \max } p(r, s) \\
& =\underset{s \in \mathcal{C}}{\arg \min } d^{2}(r, s),
\end{aligned}
$$

where $d$ is the $M L$ premetric defined by

$$
\begin{aligned}
d^{2}(r, s)=\frac{\left(r_{\rho}-s_{\rho}\right)^{2}}{\sigma_{n}^{2}}+\frac{\left(r_{\theta}-s_{\theta}\right)^{2}}{\sigma_{p}^{2}+\sigma_{n}^{2} / s_{\rho}^{2}} & \\
& +\log \left(1+\frac{1}{\sigma_{p}^{2}} \cdot \frac{\sigma_{n}^{2}}{s_{\rho}^{2}}\right) .
\end{aligned}
$$

The ML premetric has proved to yield significant gain in performance compared to the Euclidean distance detector [2]
This decision rule is the minimization of the Mahalanobis distance [6]. However, in this case the covariance matrix is defined upon the amplitude of the sent symbol $s_{\rho}$. Therefore, considering $d$ is not convenient since it does not define a metric. Indeed, the map $d: \mathbb{C} \times \mathbb{C} \mapsto \mathbb{R}^{+}$is neither symmetric nor subadditive - even if the log term is considered negligble in the decision.

Without loss of generality, we can assume that $\sigma_{p}^{2}+\sigma_{n}^{2} / s_{\rho}^{2} \simeq$ $\sigma_{p}^{2}+\sigma_{n}^{2} / E_{s}$, which is a tight approximation at high SNR. This leads to the polar metric decision rule

$$
\begin{gathered}
\hat{s}=\underset{s \in \mathcal{C}}{\arg \min } d_{\gamma}^{2}(r, s), \\
d_{\gamma}^{2}(r, s)=\left(r_{\rho}-s_{\rho}\right)^{2}+\frac{\left(r_{\theta}-s_{\theta}\right)^{2}}{\gamma^{2}},
\end{gathered}
$$

where $\gamma^{2}=\sigma_{p}^{2} / \sigma_{n}^{2}+1 / E_{s}$. One of the advantages of exploiting the polar metric rather than the ML premetric is that distances are simpler to evaluate on practical systems. Besides, in doing so, the polar metric $d_{\gamma}$ is well-defined and enables us to pursue the analysis within the scope of complete metric space.

\section{B. Framework}

Lemma 1. Let the complex number $s$ be represented by its polar coordinates $\left(s_{\rho}, s_{\theta}\right) \in \mathbb{P}=[0, \infty) \times[-\pi, \pi]$. Then the space $\mathbb{P}^{N}$ - of sequences $\boldsymbol{s}=\left\{s_{\rho, k}, s_{\theta, k}\right\}_{1 \leq k \leq N}$ of $N$ numbers in $\mathbb{P}$ - is a complete metric space when equipped with the metric $d_{\gamma}: \mathbb{P}^{N} \times \mathbb{P}^{N} \mapsto \mathbb{R}^{+}$defined by

$$
d_{\gamma}^{2}(\boldsymbol{x}, \boldsymbol{y})=\sum_{k=1}^{N}\left(x_{\rho, k}-y_{\rho, k}\right)^{2}+\frac{\left(x_{\theta, k}-y_{\theta, k}\right)^{2}}{\gamma^{2}}, \quad \gamma^{2} \in \mathbb{R}_{*}^{+},
$$

for every $\boldsymbol{x}, \boldsymbol{y}$ vectors of $\mathbb{P}^{N}$.

Proof: It is sufficient to demonstrate that $\mathbb{P}^{N}$ is a closed subset of the complete metric space $\left(\mathbb{R}^{2 N}, d_{\gamma}\right)$. First, it is trivial that the map $d_{\gamma}$ is a weighted Euclidean distance on $\mathbb{R}^{2 N}$. It follows immediately that $\left(\mathbb{R}^{2 N}, d_{\gamma}\right)$ is a complete metric space. Ultimately, the metric space $\left(\mathbb{P}^{N}, d_{\gamma}\right)$ is complete as $\mathbb{P}^{N}$ is clearly a closed subset of $\mathbb{R}^{2 N}$.

From now on, we will abbreviate by $p_{2}$ the metric space $\left(\mathbb{P}^{N}, d_{\gamma}\right)$ providing the framework to study the Gaussian PN channel. Metric spaces, by generalizing the notion of distances, provide a fundamental framework for telecommunications. Lemma 1 ensure that efficient algorithms may be designed for the demodulation of signals over the Gaussian PN channel. By way of example, the Nearest Neighbor (NN) search to realize the symbol detection in Eq. (10) or to compute the probabilistic demapper values later defined in Eq. (17) - may be performed very efficiently within $p_{2}$ using projections or sphere decoding. However, design of such algorithm exceeds the scope of this paper and is to be addressed in a future dedicated one.

To conclude, it is important to highlight the strong similarity of the Gaussian PN channel and the complex AWGN one. This 


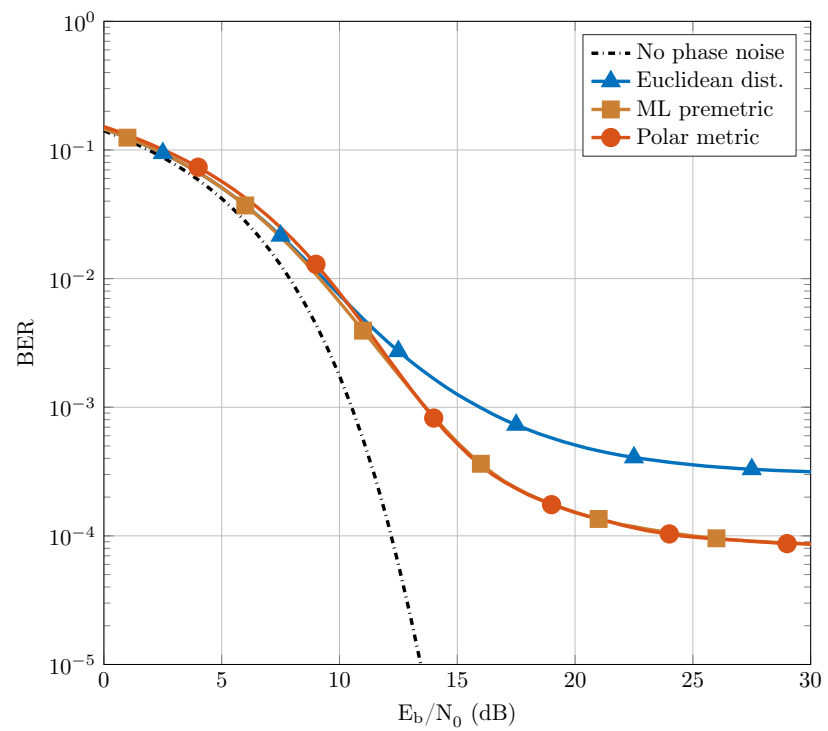

Fig. 1: Performance of the different decision rules for an uncoded 16-QAM with PN variance $\sigma_{p}^{2}=10^{-2}$

is well illustrated by the fact that the system model in Eq. (1) and (2) can be approximated within $\mathbb{P}$ by

$$
\left(r_{\rho}, r_{\theta}\right) \simeq\left(s_{\rho}+n_{\rho}, s_{\theta}+n_{\theta}\right),
$$

where $n_{\rho}$ and $n_{\theta}$ follows Gaussian distributions with respect to Eq. (6). In fact, we entitled the space $p_{2}$ by analogy to the space $l_{2}{ }^{1}$, usually exploited to study discrete signals over linear channels.

\section{Application: demodulation of uncoded M-QAM}

We depict in Fig. 1 the BER performance of an uncoded 16QAM with detection based either on the Euclidean distance, or on the ML premetric, or on the polar metric. First, it should be highlighted that the error floor, due to PN, is significantly reduced by using an appropriate decision rule. These simulations also demonstrate that performance achieved by the polar metric, with a simpler expression, are identical to the ML premetric one at high SNR. Fig. 1 is an illustration of the performance maximization through Channel-State-Information (CSI) capitalization. It is worth mentioning that using channel statistics to properly represent the signal and hence optimize the communication performance is a well-known scheme, implemented for instance in the Orthogonal Time Frequency Space (OTFS) waveform [7].

\section{Probabilistic Demapper}

Channel coding is indivisible of modern communication systems, for it provides a means to achieve robust communications over noisy channels. Furthermore, it is usually complemented with soft decision decoding, which is known to improve significantly the performance of channel decoders.

\footnotetext{
${ }^{1}$ The set of complex-valued sequences equipped with the Euclidean inner product
}

Namely, soft decision decoding exploits the reliability information of the encoded bits. Nevertheless, it may be too complex for practical systems to evaluate directly the exact values of a probabilistic demapper. Still, they can be closely approximated in order to be efficiently computed.

\section{A. Bit LLR values}

Let us here consider a Bit-Interlaved-Coded-Modulation (BICM) architecture such that the symbol $s$ maps the binary word $\boldsymbol{b}=\left(b_{1}, \ldots, b_{\log _{2}(M)}\right)$. The optimum hard decision on the simple detection problem regarding bit $b_{i}$ is achieved by the ML criterion [6]

$$
\hat{b}_{i}(r)=\left\{\begin{array}{ll}
1, & \text { if } L_{i}(r)>0 \\
0, & \text { otherwise }
\end{array},\right.
$$

where $L_{i}$, the Log-Likelihood-Ratio (LLR) of decision $\hat{b}_{i}$, is defined by

$$
L_{i}(r)=\log \left(\frac{p\left(b_{i}=1 \mid r\right)}{p\left(b_{i}=0 \mid r\right)}\right) .
$$

While the sign of the LLR supplies the decision to be taken, its absolute value quantifies the reliability of this decision. By applying Bayes' rule and assuming equiprobable symbols, the LLR may be written as

$$
L_{i}(r)=\log \left(\frac{\sum_{s_{1} \in \mathcal{C}_{1}^{i}} p\left(r \mid s_{1}\right)}{\sum_{s_{0} \in \mathcal{C}_{0}^{i}} p\left(r \mid s_{0}\right)}\right),
$$

where $\mathcal{C}_{\beta}^{i}$ denote the subset of the constellation symbols satisfying $b_{i}=\beta$. This expression are tedious to evaluate directly. However, simplified but suboptimal LLR can be obtained through the commonly used max-log sum approximation [8], viz. $\log \left(\sum_{k} x_{k}\right) \simeq \max _{k} \log \left(x_{k}\right)$. This approximation is tight at high SNR and leads to

$$
L_{i}(r) \simeq \max _{s_{1} \in \mathcal{C}_{1}^{i}} \log \left(p\left(r \mid s_{1}\right)\right)-\max _{s_{0} \in \mathcal{C}_{0}^{i}} \log \left(p\left(r \mid s_{0}\right)\right) .
$$

In addition, most of implemented decoder algorithms are not sensitive to normalization, e.g. the min-sum decoder for LDPC. Such that, we can express the LLR upon the polar metric defined in Eq. (10) by

$$
L_{i}(r) \simeq \min _{s_{0} \in \mathcal{C}_{0}^{i}} d_{\gamma}^{2}\left(r, s_{0}\right)-\min _{s_{1} \in \mathcal{C}_{1}^{i}} d_{\gamma}^{2}\left(r, s_{1}\right) .
$$

Several comments should be pointed out. First, this expression is identical to the LLR of an AWGN channel if $d$ denoted the Euclidean distance [8]. Furthermore, the bit LLR values in Eq. (17) can be computed simply - which is not the case for the ML premetric - since the polar metric $d_{\gamma}$ is no more than a weighted Euclidean distance within $p_{2}$. Although the proposed demodulation is applied to QAM constellation in the following, it is valid for any modulation scheme. 


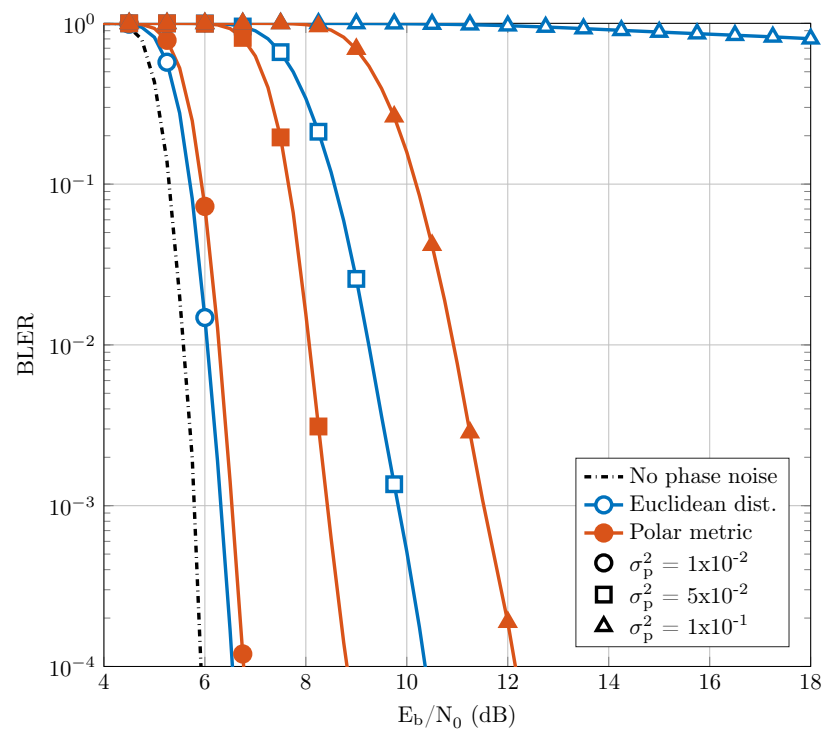

Fig. 2: Comparison of a LDPC coded 16-QAM performance exploiting LLR based either upon the Euclidean distance or on the polar metric for different values of PN variance $\sigma_{p}^{2}$

\section{B. Application: demodulation of LDPC coded $M-Q A M$}

To the best of our knowledge, performance gains provided by an appropriate decision metric for demodulation of coded systems over the Gaussian PN channel have never been evaluated. Besides, in the high-rate communications context, Low-Density-Parity-Check (LDPC) codes are recognized as a foremost solution for error-correcting codes. Due to their excellent performance and highly parallelizable decoders, they have been adopted in several recent communication standards, e.g. 5G-NR [9]. Therefore, a LDPC code appears as the most sensible choice to evaluate the proposed LLR expression. To be specific, the implemented code follows the specifications of the IEEE 802.11 standard [10] with a min-sum decoder, a block length of 1944 bits and a coding rate of 2/3. Fig. 2 presents the BLock Error Rate (BLER) performance of a 16-QAM for arbitrary values of PN variance. In particular, it is shown that the polar metric yields significant gain in performance compared to the Euclidean distance. If the SNR is low and the PN variance is minor, then the Euclidean detector slightly outperforms $(<0.1 \mathrm{~dB})$ the polar metric demodulation - as expected since the polar metric relies on the high SNR assumption. Yet, as PN gets stronger, the performance gain becomes more and more significant. Eventually for strong PN scenarios, the polar metric is the only one exhibiting the waterfall feature of LDPC, while the iterative decoder never converges with the Euclidean distance. Performance of the ML premetric are not shown as they are strictly identical to the ones of the polar metric, even though they are more complex to evaluate.

\section{Channel estimation For Optimal Demodulation}

In previous sections, it has been emphasized that knowledge of the CSI is highly valuable to maximize the performance of communication systems affected by PN. Conversely to the
Euclidean distance, the ML premetric and the polar metric are directly defined upon the thermal and phase noise variances. Greatly improving the performance, both decisions rules hence require an estimation of the values $\sigma_{n}^{2}$ and $\sigma_{p}^{2}$.

\section{A. Thermal and phase noise variances estimation}

Estimation of thermal and phase noise variances $\sigma_{n}^{2}, \sigma_{p}^{2}$ can be performed by transmitting $N$ pilot symbols. These pilot samples are known from the receiver and denoted $s=$ $\left(s_{1}, s_{2}, \ldots, s_{N}\right)$. The joint likelihood function may be derived from Eq. (7) and is expressed by

$$
p_{N}\left(\boldsymbol{r} \mid s, \sigma_{n}^{2}, \sigma_{p}^{2}\right)=\prod_{k=1}^{N} p\left(r_{k} \mid s_{k}, \sigma_{n}^{2}, \sigma_{p}^{2}\right)
$$

where $\boldsymbol{r}=\left(r_{1}, \ldots, r_{N}\right)$ stands for the received samples. We consider in the following that pilots have a constant amplitude ${ }^{2}$ of $\sqrt{E_{s}}$. The ML estimate [6] of the covariance matrix yields the ML estimators expressed by

$$
\begin{aligned}
& \hat{\sigma}_{n}^{2}=\frac{1}{N} \sum_{k=1}^{N}\left(r_{\rho, k}-\sqrt{E_{s}}\right)^{2}, \\
& \hat{\sigma}_{p}^{2}=\frac{1}{N} \sum_{k=1}^{N}\left(r_{\theta, k}-s_{\theta, k}\right)^{2}-\frac{\hat{\sigma}_{n}^{2}}{E_{s}} .
\end{aligned}
$$

We can write such estimators as $\chi^{2}(N)$ distributions to quantify their bias and dispersion from the means and variances:

$$
\left\{\begin{array}{l}
\mathrm{E}\left[\hat{\sigma}_{n}^{2}\right]=\sigma_{n}^{2} \\
\mathrm{~V}\left[\hat{\sigma}_{n}^{2}\right]=\frac{2 \sigma_{n}^{4}}{N}
\end{array} \quad, \quad\left\{\begin{array}{l}
\mathrm{E}\left[\hat{\sigma}_{p}^{2}\right]=\sigma_{p}^{2} \\
\mathrm{~V}\left[\hat{\sigma}_{p}^{2}\right]=\frac{2\left(\sigma_{p}^{2}+\sigma_{n}^{2} / E_{s}\right)^{2}}{N}+\frac{2 \sigma_{n}^{4}}{N E_{s}}
\end{array} .\right.\right.
$$

Both of these estimators are unbiased. Therefore, it is relevant to compare their dispersions to the Cramer-Rao Lower Bounds (CRLB) [6]. Since Eq. (18) satisfies the regularity conditions, the CRLBs are given by

$$
\mathrm{V}\left[\hat{\sigma}_{n}^{2}\right] \geq \frac{2 \sigma_{n}^{4}}{N}, \quad \mathrm{~V}\left[\hat{\sigma}_{p}^{2}\right] \geq \frac{2\left(\sigma_{p}^{2}+\sigma_{n}^{2} / E_{s}\right)^{2}}{N} .
$$

Since the variance of $\hat{\sigma}_{n}^{2}$ equals the CRLB, the estimator is efficient $c f$. [6]. Regarding the PN variance, the estimator $\hat{\sigma}_{p}^{2}$ is close to the CRLB but does not perfectly achieves it. Still, both estimators demonstrate the smallest Mean Square Error (MSE) among any unbiased estimators. From Eq. (18), it is straightforward that the joint density $p_{N}$ belongs to the exponential family such that $T=\left(\sum_{k=1}^{N}\left(r_{\rho, k}-\sqrt{E_{s}}\right)^{2}, \sum_{k=1}^{N}\left(r_{\theta, k}-s_{\theta, k}\right)^{2}\right)$ is a complete sufficient statistic for parameter $\left(\sigma_{n}^{2}, \sigma_{p}^{2}\right)$. By the Lehman-Scheffé theorem, the unbiased estimators $\left(\hat{\sigma}_{n}^{2}, \hat{\sigma}_{p}^{2}\right)$, defined upon $T$, are respectively the unique MinimumVariance Unbiased Estimators (MVUE) of $\sigma_{n}^{2}$ and $\sigma_{p}^{2}$.

\footnotetext{
${ }^{2}$ The average symbol energy $E_{s}$ is considered perfectly known as it can be estimated blindly through all sent symbols.
} 


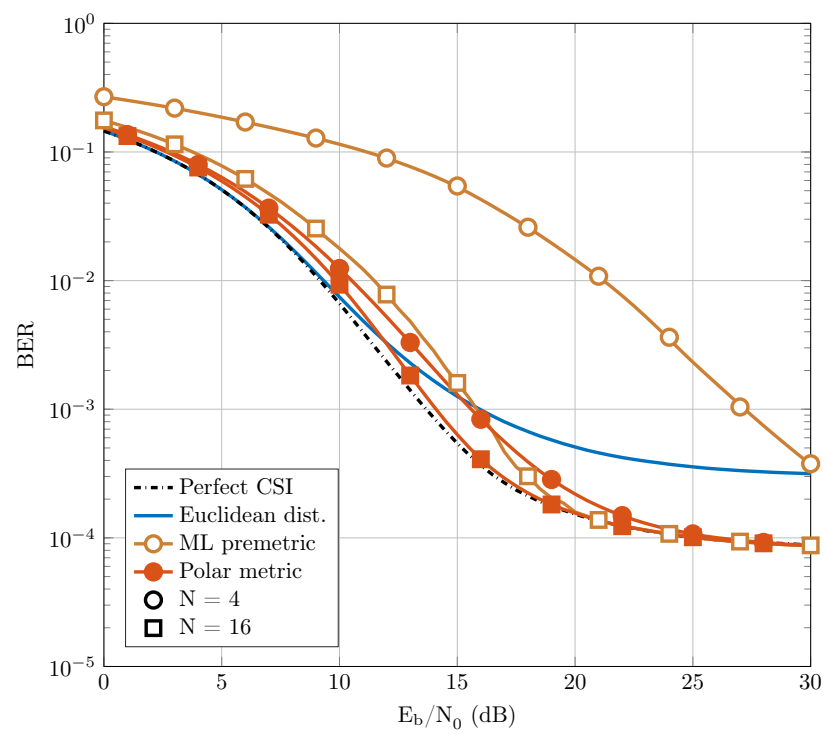

Fig. 3: Performance degradation due to estimation errors for an uncoded 16-QAM and different number of pilot samples with PN variance $\sigma_{p}^{2}=10^{-2}$

\section{B. Performance degradation due to estimation errors}

Though we have been able to evaluate the performance of the estimators. Yet, definitions of the ML premetric and the polar metric are subject to estimation errors. So that, the performance degradation due to such estimation errors has to be quantified to specify the required number of pilots. Therefore, Fig. 3 and 4 present respectively the performance of an uncoded and a LDPC coded 16-QAM for different number of pilots symbols. We may remark that the polar metric is significantly more resilient than the ML premetric to estimation errors for hard symbol detection, and only slightly in the case of soft decision decoding. A rather low number of pilots is required to achieve near optimal performance.

\section{CONCLUSION}

In this paper, we have addressed the problem of demodulation in the presence of PN. We have assumed a high SNR and a Gaussian PN to derive the optimum symbol detection decision rule. This ML decision premetric significantly reduces the BER error floor due to PN. Besides, it is possible to define a simpler metric, entitled polar metric, upon this decision rule without any loss in performance. Thereupon, we have proposed a framework to properly represent signals upon the Gaussian PN channel. Further, computable probabilistic demapper values have been defined upon the polar metric, which is not direct from the ML premetric. It has been shown that the polar metric, in comparison to the Euclidean distance, greatly enhances performance of coded systems. Since optimum demodulation requires the knowledge of CSI, we have proposed ML estimates of the thermal and phase noise variances. By evaluating the performance degradation due to estimation errors, we are able to state that a low number of pilots is sufficient to achieve near optimal performance. It is also worth mentioning that these results could be extented to non-mmWave applications and Rayleigh fading channels.

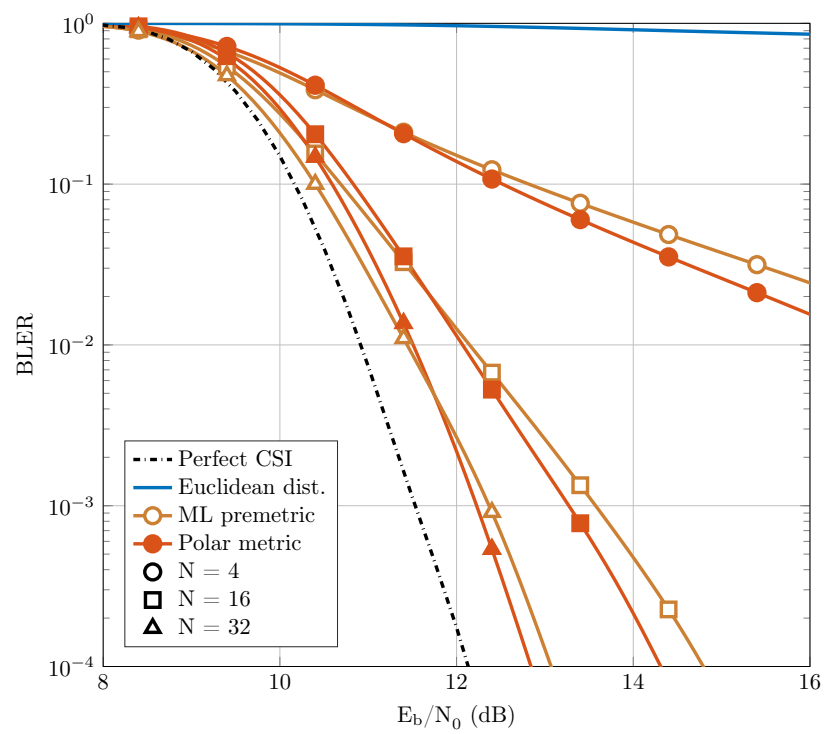

Fig. 4: Performa.nce degradation due to estimation errors for a LDPC coded 16-QAM and different number of pilot samples with PN variance $\sigma_{p}^{2}=10^{-1}$

\section{ACKNOWLEDGMENT}

The research leading to these results received funding from the French National Research Agency (ANR-17-CE25-0013) within the frame of the project BRAVE.

\section{REFERENCES}

[1] M. Voicu, D. Pepe, and D. Zito, "Performance and Trends in MillimetreWave CMOS Oscillators for Emerging Wireless Applications," International Journal of Microwave Science and Technology, vol. 2013, p. 6, 2013.

[2] R. Krishnan, M. R. Khanzadi, T. Eriksson, and T. Svensson, "Soft metrics and their performance analysis for optimal data detection in the presence of strong oscillator phase noise," CoRR, vol. abs/1310.1638, 2013.

3] R. Krishnan, A. G. i Amat, T. Eriksson, and G. Colavolpe, "Constellation Optimization in the Presence of Strong Phase Noise," IEEE Transaction on Communications, vol. 61, no. 12, pp. 5056-5066, December 2013.

[4] M. R. Khanzadi, D. Kuylenstierna, A. Panahi, T. Eriksson, and H. Zirath, "Calculation of the Performance of Communication Systems From Measured Oscillator Phase Noise," IEEE Transactions on Circuits and Systems I: Regular Papers, vol. 61, no. 5, pp. 1553-1565, May 2014.

[5] J. Proakis, Digital Communications 5th Edition, ser. McGraw-Hill series in electrical and computer engineering : communications and signal processing. McGraw-Hill, 2007.

[6] T. Moon and W. Stirling, Mathematical Methods and Algorithms for Signal Processing. Prentice Hall, 2000.

[7] R. Hadani, S. Rakib, M. Tsatsanis, A. Monk, A. J. Goldsmith, A. F. Molisch, and R. Calderbank, "Orthogonal Time Frequency Space Modulation," in 2017 IEEE Wireless Communications and Networking Conference (WCNC), March 2017, pp. 1-6.

[8] F. Tosato and P. Bisaglia, "Simplified soft-output demapper for binary interleaved COFDM with application to HIPERLAN/2," in 2002 IEEE International Conference on Communications. Conference Proceedings. ICC 2002 (Cat. No.02CH37333), vol. 2, 2002, pp. 664-668 vol.2.

[9] 3rd Generation Partnership Project (3GPP), "Overall Description Stage 2," Technical Specification Group Access Network 38.300, 122017 , 20.0

[10] IEEE Computer Society, "IEEE Standard for Information technologyLocal and metropolitan area networks- Specific requirements- Part 11: Wireless LAN Medium Access Control (MAC)and Physical Layer (PHY) Specifications Amendment 5: Enhancements for Higher Throughput," IEEE Std 802.11n-2009 (Amendment to IEEE Std 802.11-2007 as amended by IEEE Std 802.11k-2008, IEEE Std 802.11r-2008, IEEE Std 802.11y-2008, and IEEE Std 802.11w-2009), pp. 1-565, Oct 2009. 